\title{
Nutritional Stress of Cultured Vero Cells Causes Altered Growth and Morphology as Seen in Neoplastic Transformation
}

\author{
Tyler Adams, Rabia Anwar, Michael Mfarej, Taylor Rundatz, \\ Melissa Coyle, and Dr. Jacqueline S. McLaughlin \\ Department of Biology, Penn State University Lehigh Valley, Center Valley, PA \\ Students: tadams1845@gmail.com,ranwar997@gmail.com,mfarejm@yahoo.com, \\ taylorrundatz4@gmail.com \\ Mentors: jxm57@psu.edu,msg110@psu.edu
}

\begin{abstract}
In this report, the authors describe the effects of stressed culture conditions on Vero cells, which are a form of epithelial cells derived from the African green monkey kidney. This project was designed in large part to replicate a previously published report on the effects of nutritional stress on the growth patterns of these cells. Culture conditions that include nutritional limitation and cell crowding have been shown to transform these cells into cells that differ morphologically, develop into spheroid-shaped clusters, as well as exhibit altered protein expression. It was suggested that these changes might mirror those occurring in metastatic cancer cells, and could therefore provide a useful experimental model system. In the current study, the investigators successfully cultured Vero cells in control cultures, and then compared their growth patterns and morphology to cells grown in nutritionally stressed and overcrowded cell culture conditions defined by extensive days in culture in unchanged media which did or did not contain glucose. The results confirmed that nutritionally stressed and over-crowded cultures result in cells that change morphologically, detach from the substrate, and exhibit spheroid-shaped clusters of cells. Sub-culturing these detached cells demonstrated that the results were permanent, meaning that the new growth patterns and clustering persisted. The results suggest that deprivation of nutrition and other factors essential to life may facilitate aberrant growth.
\end{abstract}

\section{KEYWORDS}

Vero Cells, Nutritional Stress, Cell Morphology, Neoplastic Transformation, Malignant Cells, Spheroid Formation.

\section{INTRODUCTION}

Vero cells are epithelial cells derived from the kidney of an African green monkey (Cercopithecus aethiops), which can be used for many purposes including: screening for the toxin of Escherichia coli; as host cells for growing specific viruses; or, as host cells for eukaryotic parasites. The Vero cell lineage was developed in 1962 by Yasumura and Kawakita at Chiba University in Chiba, Japan. Vero cells have a distinctive growth pattern in culture. The cells of this lineage grow in monolayers, are elongated in shape, and are similar to fibroblast cells with little cytoplasmic granulation. When a Vero cell population reaches cellular confluence, sub-culture should be carried out; otherwise, the cells degenerate or lift off the culture flask surface. ${ }^{1}$

Transformation is a multistep process that often culminates in the production of neoplastic (malignant) cells. ${ }^{2}$ Fibroblasts and epithelial cells have been shown to undergo neoplastic transformation in cell culture. According to Smets, cellular transformation itself involves a modification or serial modifications in the cellular genome, which lead to an overall altered phenotype. ${ }^{3}$ Morphological changes can be seen through altered growth and adhesion and spreading characteristics. ${ }^{4-6}$ Importantly, cellular transformation can be induced in vitro by several factors such as viruses, 
chemicals, radiation and metabolic injury, and as such, transformed cells have been used to study processes comparable to neoplastic transformation in vivo to better understand the origin and progression of tumors in animals. ${ }^{7-9}$ The interest here focuses on cellular transformation of Vero cells that is induced by nutritional stress and over-crowding.

Vero cells have also been shown to be a model system for studying nutritional stress-induced cellular transformation in vitro. If grown to confluence and left without media change for up to 15 days, nutritionally stressed Vero cells exhibit multilayered growth with many cells either detaching and growing freely in suspension or aggregating into cellular clusters called "spheroids". ${ }^{10}$ Normal fibroblasts or epithelial cells require adhesion and spreading on the substrate for growth in vitro, while several tumor cell lines present the ability to grow in suspension. ${ }^{11}$ Spheroids have been extensively characterized in cancer cells and embryonic cells ${ }^{12-14}$ and numerous research gives evidence that malignant cells of solid tumors, fetal cells of various organs, and liver cells of newborn rats aggregate and form spheroids when they are cultured on non-adhesive substrata. ${ }^{13}$ Additionally, cells participating in spheroid formation exhibit decreased glucose consumption, lactic acid production, and ATP content compared to cells in a monolayer, i.e., anaerobic metabolism ${ }^{14}$ and reveal higher polyploidy index than non-spheroidal cells. ${ }^{1}$

The goal of this experiment was to provide further evidence that Vero cell transformation is inducible in cell culture by engineering culture conditions with, and without, nutritional stress and overcrowding. Vero cells were grown over both nine day and fifteen day periods under the following conditions: control group with glucose and media change; experimental group with glucose and no media change; and, experimental group without glucose and no media change. Cell growth patterns and morphology were extensively monitored over this time period on a daily basis. For the fifteen day study, supernatant, which included spontaneously detached cells and spheroids, was collected and placed in culture flasks for an additional seven day period. The overall results substantiate that cell starvation and over-crowding in culture results in cells that detach from the substrate and/or exhibit spheroid-shaped clusters of cells. Additionally, the sub-culturing of these cells demonstrated that these morphological changes and behaviors are permanent, meaning that free-floating cells and spheroidal clusters persisted and were viable.

\section{METHODS AND MATERIALS}

\section{A. Control Culture Conditions}

Vero cells, obtained from Penn State York campus, were maintained in Roswell Park Memorial Institute (RPMI) 1640 media containing $2 \mathrm{~g} / \mathrm{L}$ glucose (Cellgro, 10-040-CM) supplemented with 5\% fetal bovine serum (FBS; Cellgro, 35-010-CV) and 1\% penicillin-streptomycin (Hyclone, SV30010). The cells were stored at $37^{\circ} \mathrm{C}$ with $5 \% \mathrm{CO}_{2}$ (ThermoForma Series II). The cells maintained under these conditions were known as the "control glucose" group (CG) since the media was replenished every 2-3 days. Sub-culture was not performed at confluence of the monolayers, and two groups of cultures were maintained for both nine and fifteen days (Figure 1). For the nine day experiment, ten T-25 flasks of cells were maintained for the CG group (Figure 1A) and were analyzed on a daily basis for cell confluency and morphology (Figure 1A). Importantly, one flask from this group was selected on each day of experimentation for analysis of cell count and viability. For the fifteen day experiment, fifteen T-25 flasks were maintained for the CG group and flasks were analyzed on a daily basis for cell confluency and morphology. Cell counts and viability were obtained from CG flasks on day 15 only, and both the adhered cells and culture supernatant were analyzed (Figure 1B).

\section{B. Experimental Culture Conditions}

Two nutritionally stressed and overcrowded experimental groups were created: "experimental glucose" (EG) and "experimental no glucose" (ENG). Both groups were maintained without change of culture media for both nine and fifteen days. These time spans were chosen as changes in growth 
characteristics were not observed over shorter periods of time, and most cell cultures presented senescence or death in cultures over 15 days - as per the work of Genari et al. (1998). ${ }^{10}$ EG cells were grown to confluence in RPMI 1640 containing $2 \mathrm{~g} / \mathrm{L}$ glucose (Cellgro, 10-040-CV) supplemented with 5\% FBS (Cellgro, 35-010-CV) and 1\% penicillin-streptomycin (Hyclone, SV30010) in $37^{\circ} \mathrm{C}$ with $5 \% \mathrm{CO}_{2}$. ENG cells were maintained in the same growth media, RPMI 1640, but contained no glucose (Cellgro, 10-043-CV) supplemented with 5\% FBS (Cellgro, 35-010-CV) and 1\% penicillin-streptomycin (Hyclone, SV30010). For the nine day experiment, ten T-25 flasks of cells were maintained for each of the EG and ENG groups (Figure 1A) and were analyzed for confluency and morphology on a daily basis. Importantly, one flask from this group was selected on each day of experimentation for analysis of cell count and viability. For the fifteen day experiment, fifteen T-25 flasks were maintained for each of the EG and ENG groups and were analyzed on a daily basis for cell confluency and morphology. Importantly, cell counts and viability were obtained from EG and ENG flasks on day 15 only, and both adhered cells and culture supernatant were analyzed (Figure 1B).

A.

\begin{tabular}{|c|c|c|c|c|c|c|c|c|c|}
\hline \multirow[b]{3}{*}{ CG } & & & & & Days & & & & \\
\hline & 1 & 2 & 3 & 4 & $\overline{55}$ & 6 & 7 & 8 & 9 \\
\hline & & & & & & & & & \\
\hline EG & & & & & & & & & \\
\hline NG & & & & & & & & & \\
\hline
\end{tabular}

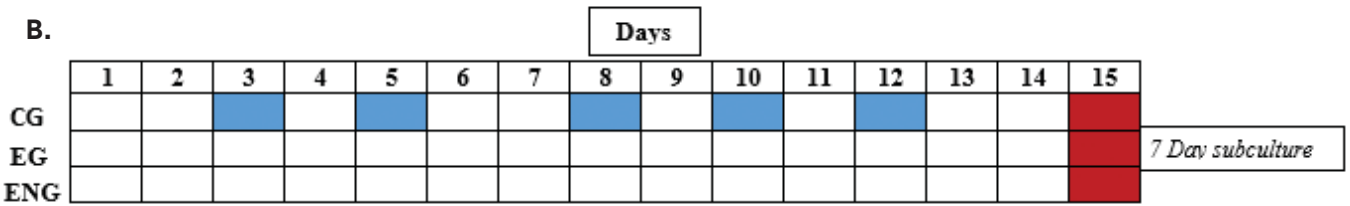

Figure 1. Experimental design showing culture conditions for the 9 day preliminary study (A) and the subsequent 15 study (B). Media change is denoted by blue boxes in control groups (CG). Attached cells (adhered to flask) and suspended cells (free-floating in supernatant) were counted on day 15 and are represented by red boxes in CG, EG, and ENG groups. Following cell counting and viability analysis, the remaining supernatant was centrifuged, re-suspended in fresh media, re-plated, and monitored microscopically for an additional seven days. No media changes were performed on these additional seven days.

\section{Cell Plating}

On day 0 of both experiments, the CG, EG, and ENG cultures were prepared by transferring $5 \mathrm{~mL}$ of Vero cells at approximately $1 \times 10^{5}$ cells/mL into the above stated T-25 flasks containing respective media conditions.

\section{Confluency}

Once the cells were plated, cell confluency (percentage of cell culture space utilized by adhering cells) was monitored microscopically over either the nine or fifteen day experimental period. Confluency was determined by observing the proportion of the flask surface occupied by adhered cells.

\section{E. Photomicrography}

Throughout the experimental period photomicrographs were taken daily of all three groups, for both 9 or 15 day experiments, using an Olympus CKX41 inverted phase-contrast microscope. Photomicrographs were taken at magnifications of 40X, 100X and 200X to document changes in cell morphology. 


\section{F. Cell Counting and Viability}

For the nine day experiment, trypsinization was performed daily on one flask in order to count attached cells using a hemocytometer. For the fifteen day experiment, trypsinization was performed on day 15 flasks only in order to count attached cells using a hemocytometer. Additionally, $1 \mathrm{~mL}$ of supernatant of cells from each group's labeled flasks was aliquoted into separate microcentrifuge tubes for cell counting. For both experiments, a trypan blue exclusion dye assay was also used to determine whether cells were viable. The trypan blue test was done by adding 50uL cell sample $+50 \mathrm{uL}$ trypan blue $+400 \mathrm{uL}$ PBS and mixing well. Next, 10uL of sample was loaded into each side of a hemocytometer slide.

\section{G. Supernatant and Subculture of Detached Cells}

Remaining supernatant from the day 15 CG, EG, and ENG group flasks was collected into 15 $\mathrm{mL}$ conical tubes. Each flask was then washed two times with $2 \mathrm{~mL}$ of D-PBS $\left(\mathrm{Ca}^{2+}\right.$ and $\mathrm{Mg}^{2+}$ free $)$ and added to its corresponding conical tube. The supernatant and D-PBS were then centrifuged for 5 minutes; the resulting cell pellet was re-suspended in $5 \mathrm{~mL}$ of fresh corresponding media and was added to a new T-25 flask. Corresponding flasks were returned to the incubator for an additional seven days. This was done to monitor any reattachment and growth of aggregates and to continually inspect suspended cells.

\section{RESULTS}

A preliminary nine day study was performed using Vero cells under the following conditions: as a control group with glucose and media change (CG); an experimental group with glucose and no media change (EG); and an experimental group without glucose and no media change (ENG) (Figure 1). It was reasoned that the CG group would grow to an over-crowded monolayer, while the EG and ENG groups would show stressed morphological changes due to nutritional stress and overcrowding. CG and EG groups reached peak confluency within 2-4 days after plating, while the ENG group never reached confluency. All groups showed no spheroid formation as nutritional deprivation and/or overcrowding increased over the nine day period; but, the ENG cells did become thin, elongated and branch-like and were less confluent (Figures $\mathbf{2}$ and $\mathbf{3}$; see Figure $\mathbf{5}$ for images of normal CG cell growth). Additionally, by day 8 of this preliminary study, ENG cells began to markedly detach from the flask surface (Figure 4A, examples shown with arrows) and exhibited noteworthy added stressed conditions of cytoplasmic vacuolation and granulation (Figure 4B, arrows). The latter morphological characteristics are abnormal in Vero cells and may indicate impaired cell health. ${ }^{15}$ This prompted a subsequent experiment employing a longer culture period of 15 days as evidenced in the work of Genari et al. (1998). ${ }^{10}$ It was reasoned, that by modifying the experimental conditions to include a 15 day culture period, cell detachment and spheroid formations, known to be associated with starvation and overcrowding, would be accentuated in the herein designed EG and ENG groups. 

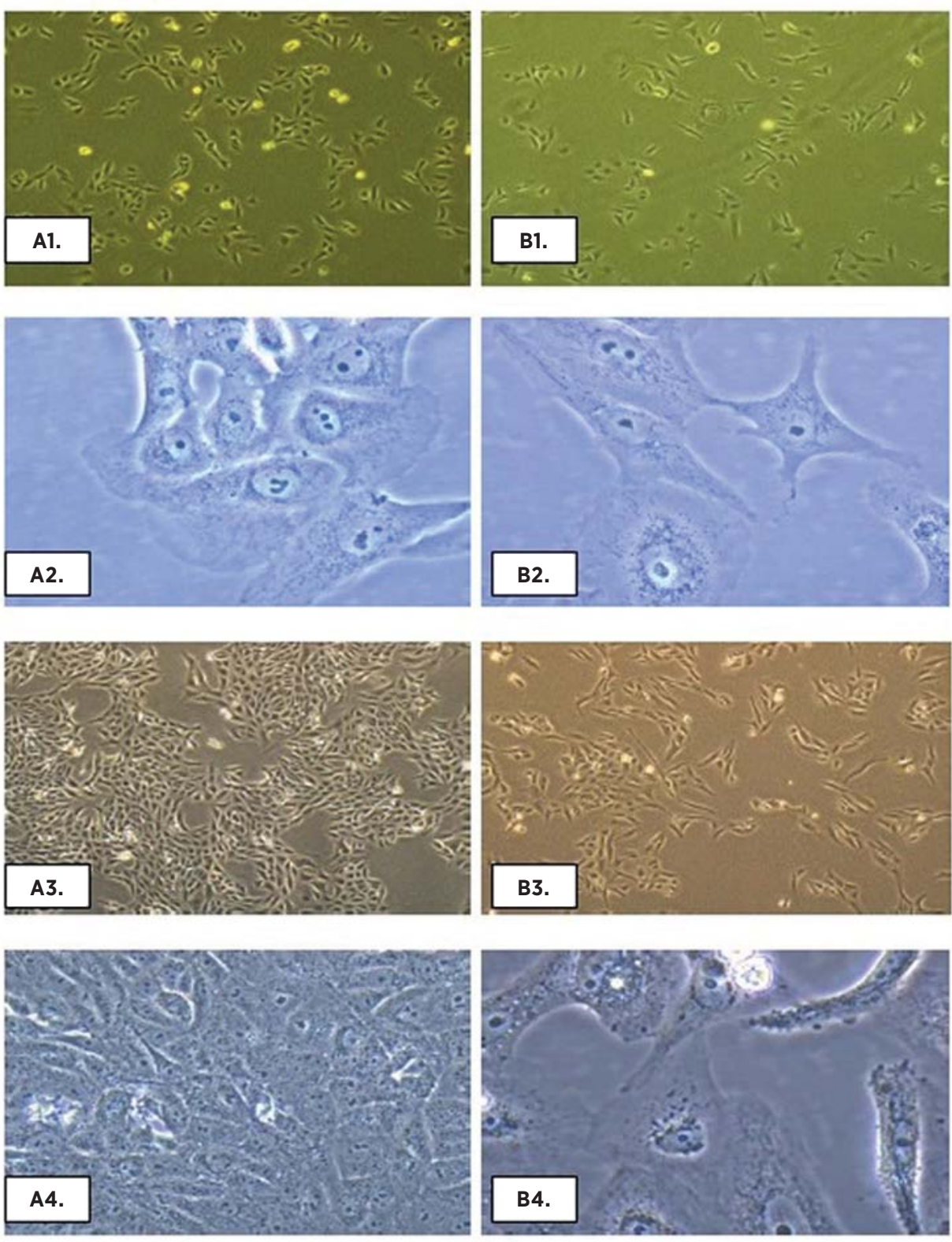

Figure 2. Photomicrographs contrasting the morphology of the EG cells. A. and the ENG cells B. from days 1-4 pilot study: A1 and B1 (40X); A2 and B2 (40X); A3 and B3 (40X); and, A4 and B4 (400X). Note elongated and thin shape in the ENG group and decreased cell confluency in comparison to EG group. 

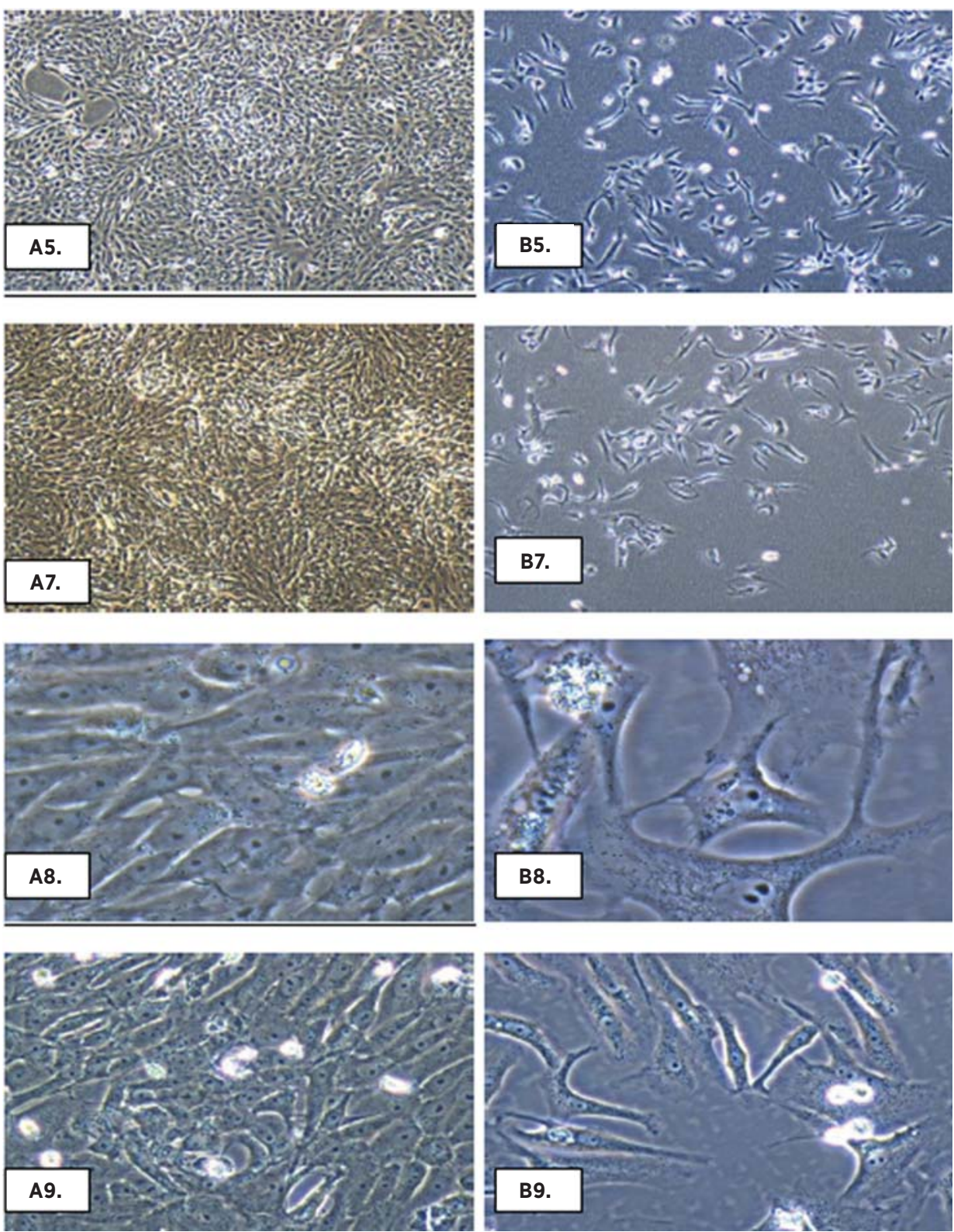

Figure 3. Photomicrographs contrasting the morphology of the EG cells (A) and the ENG cells (B) from days 5, 7, 8 and 9 pilot study: A5 and B5 (40X); A7 and B7 (40X); A8 and B8 (400X); and, A9 and B9 (200X). Note elongated and thin shape in the ENG group and decreased cell confluency in comparison to the EG group (A5-9). 


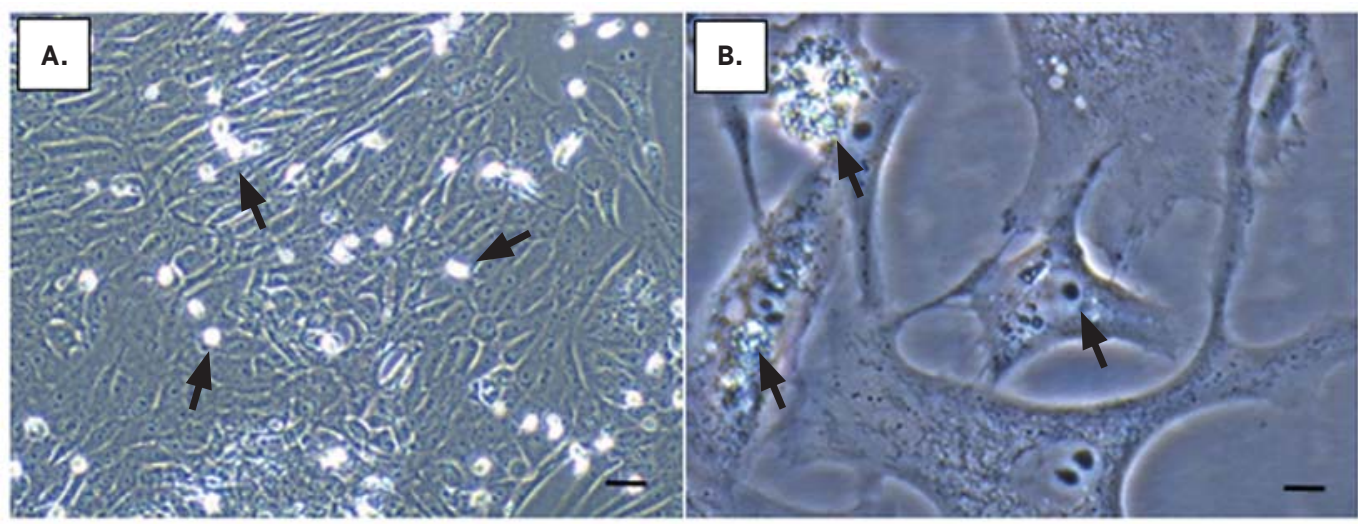

Figure 4. Images of Vero cell morphology in ENG conditions at day 8: (A) 400X (scale bar $=40 \mu \mathrm{m}$ ); and, (B) 400X (scale bar $=10 \mu \mathrm{m}$ ). Cells began to detach from the flask surface (Figure 4, arrows) and exhibited noteworthy added stressed conditions of cytoplasmic vacuolation and granulation (Figure 4B, arrows).

Similar to the pilot experiment, the CG and EG groups in the 15 day experiment reached peak confluency within 2-4 days after plating (Figure 5A-C). These two groups remained at peak confluency until days 8-9. At this time, however, the EG cells began to detach from the culture flask surface. Loss of surface adhesion was indicated by the appearance of cells that were more refractive and circular in shape as compared to adherent spindle-shaped cells. Concurrently, around day 8, the CG cells began to exhibit multi-layered growth which persisted until day 15 (Figure 5, C1 and D1). The EG cells did not exhibit the onset of multi-layered growth and remained as a confluent monolayer (Figure 5, C2 and D2). As for the ENG cells, as seen in the first experiment, confluence was never reached and cell detachment was more apparent beginning on days 8-9 and persisted until day 15 (Figure 5, C3 and D3).

As for spheroid formation, at days 13-15, EG cell growth behavior displayed the characteristic spheroid formation in culture (Figure 5, D3). Spheroidal aggregates appeared as multi-layered clusters, with non-adherent cells localized to the top surface. The cells at the peripheral edges of the spheroids were strictly elongated with diminished intercellular connections. The CG cells also displayed the formation of cell clusters at 13-15 days, but were much less concentrated and did not show the characteristics as described above. As for the ENG cell cultures on days 13-15, no spheroids were observed, and cell necrosis was apparent as cell confluence decreased and attached cells took on abnormal elongated appearances while detached cells increased in number. 


\section{Before Passage}

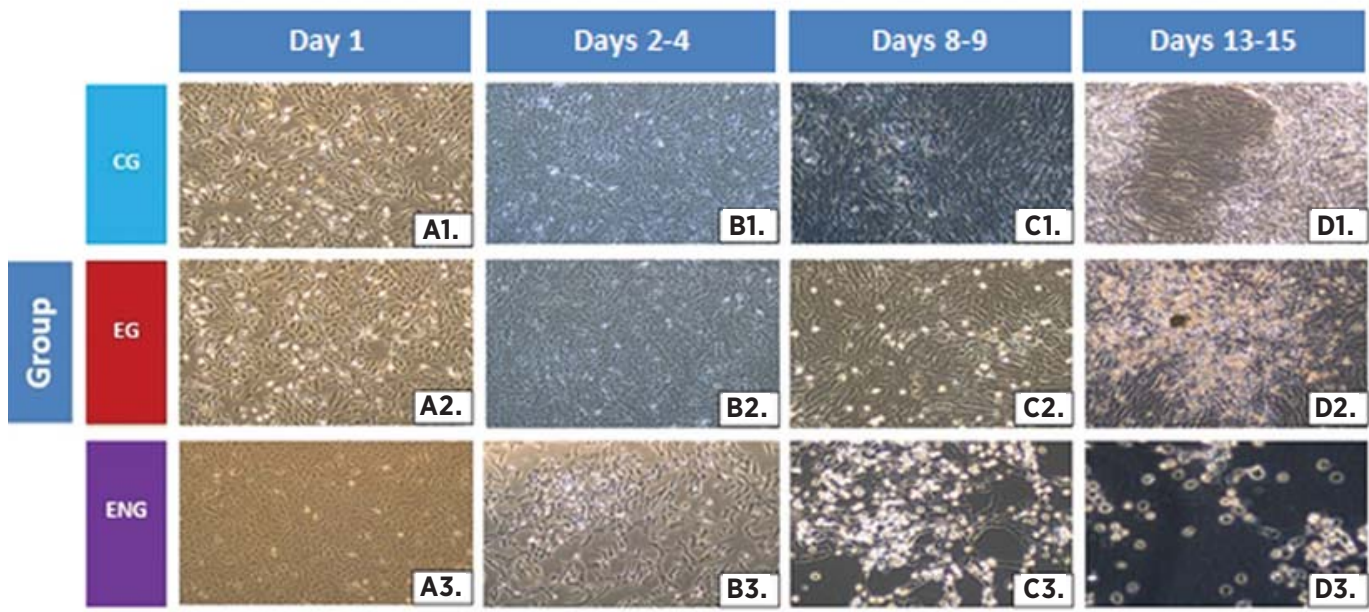

Figure 5. Photomicrographs of all groups at 40X assuming early adherence and normal morphology (A1 - A3). All groups at 40X displaying varying levels of confluency and substrate adherence (B1 - B3). All groups at 100X with EG and ENG cells exhibiting elongation and non-adherence (C1 - C3). CG and EG groups at 100X and ENG group at 200X with CG cells displaying multilayered overcrowding, EG cells with spheroid clusters, and ENG showing increased cell detachment and abnormal morphology (D1 - D3).

On day 15, cell counts and viability analysis of both the supernatant and the cells that remained attached to the flasks showed significant findings (Figure 6). The supernatant from the CG group contained very few detached cells (average $3.75 \times 10^{4}$ cells $/ \mathrm{mL}$ ); however, none of the detached cells were viable. The majority of the CG cells remained attached to the flask (average $8.05 \times 10^{6}$ cells/ $\mathrm{mL}$ ) with $99 \%$ viability. In contrast, EG cells presented an increased number of cells in the supernatant (average 1.50x10 cells $/ \mathrm{mL}$ ) with $25 \%$ of these detached cells being viable. This is essential to point out as this is the only group that presented a live detached cell population at an average concentration of $3.65 \times 10^{4}$ cells $/ \mathrm{mL}$. The portion of the EG cells which remained attached to the flask (average $6.88 \times 10^{5}$ cells $/ \mathrm{mL}$ ) were $87 \%$ viable. Lastly, while the supernatant of the ENG group confirmed observations of increased levels of cell detachment, this group also revealed the highest total cell concentration (average $2.63 \times 10^{5}$ cells $/ \mathrm{mL}$ ) in the supernatant with $0 \%$ viability. Notably, almost no cells remained attached to the flask in the ENG group (average $1.25 \times 10^{4} \mathrm{cells} / \mathrm{mL}$ ).

Since the EG group was the only group that presented live detached cells in the supernatant on day 15 , it was re-plated with fresh media and was monitored for an additional seven days. Figure 7 displays the EG group during the seven day period after passage. Within 24 hours after re-plating, the cells resumed spheroid formation (Figure 7A). Additionally, attached cells retained cytoplasmic granulation which was first evident before passage (Figure 7B). Other morphological changes noted included enhanced elongation and spindle-like cytoplasmic extensions. Moreover, seven days after sub-culturing, the cells showed aberrant plasmolytic (shrinkage or contraction of the protoplasm away from the wall of a living cell) morphology. The cells demonstrated failure to reestablish intercellular associations and normal spreading characteristics (Figure $7 \mathrm{C}$ ). In summary, abnormal morphology and growth was maintained throughout the seven days after passage, i.e., cells did not recover normal Vero cell phenotype or monolayer growth pattern. 


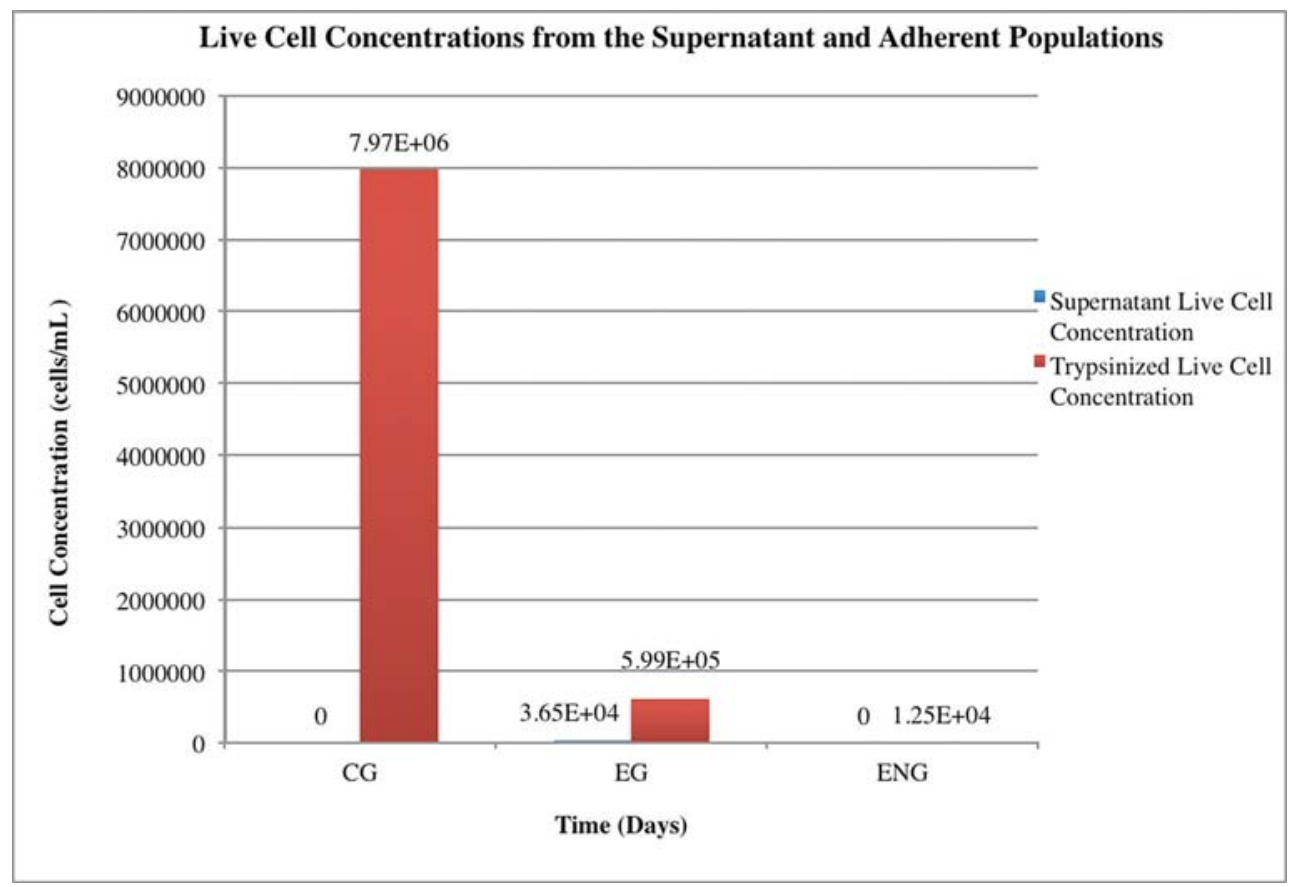

Figure 6. Histogram displaying live average cell count data taken on day 15 from the supernatant of the CG, EG, and ENG cultures (blue bars) and their trypsinized adherent cell fractions (red bars).
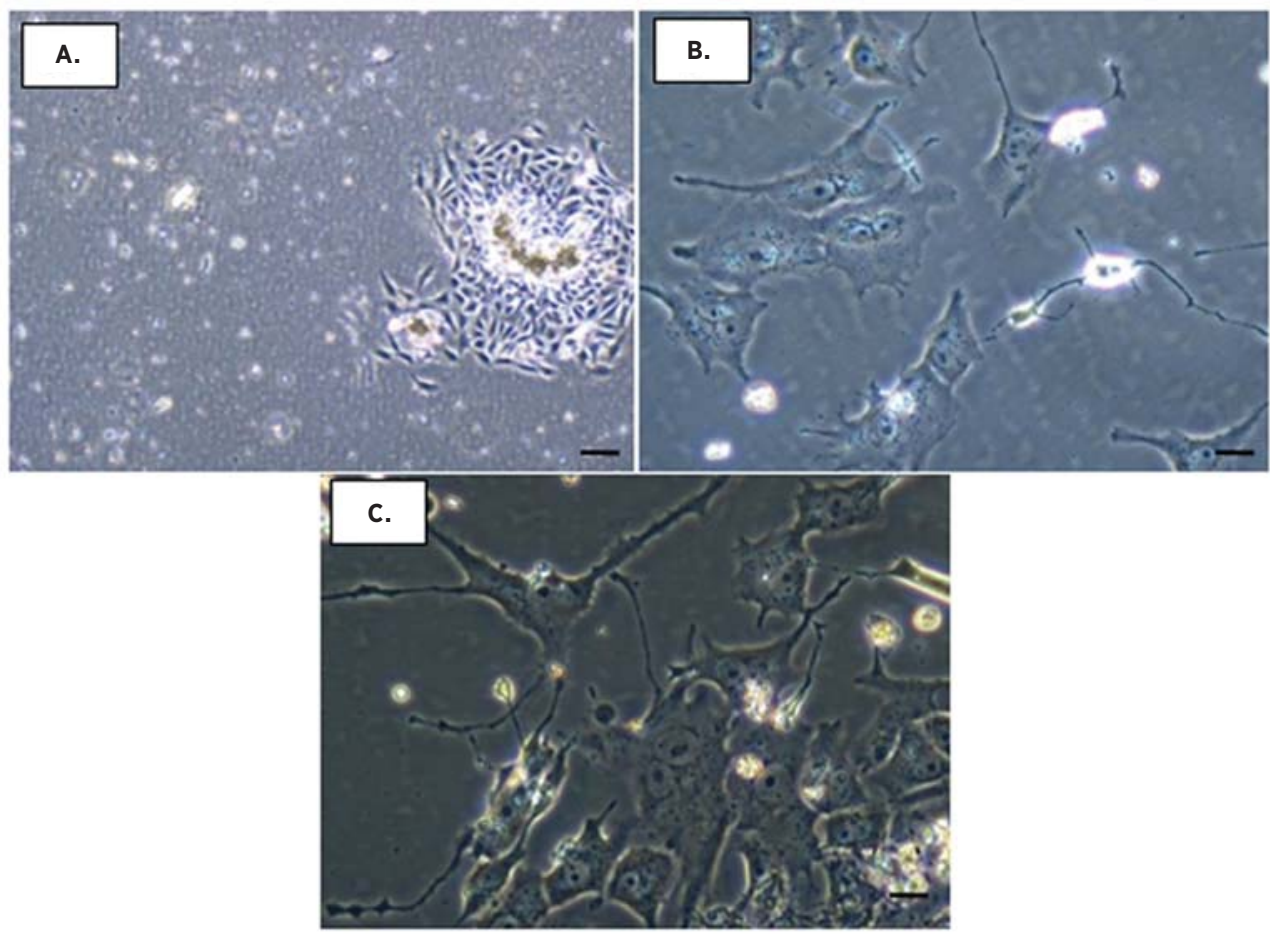

Figure 7. EG group displaying progression of abnormal cell morphology and growth characteristics after passage: A. $48 \mathrm{~h}$ (2 days) after passage showing re-adherence of transformed cells and spheroid formation (scale bar $=100 \mu \mathrm{m}$ ).

B. $144 \mathrm{~h}$ (6 days) after passage showing development of spindle-like cytoplasmic extensions (scale bar $=20 \mu \mathrm{m}$ ).

C. $168 \mathrm{~h}$ (7 days) after passage revealing prominent plasmolytic shape and reduced cell spreading (scale bar $=20 \mu \mathrm{m})$. 


\section{DISCUSSION}

The culture conditions employed in this experiment were used to further investigate previous findings on nutritionally-stressed and over-crowded Vero cells in vitro. Our results were similar, as the Vero cells grown without media change in the presence of normal media, the EG group, spontaneously detached after maintenance at peak confluency, being markedly noticeable at day 8 . This spontaneous detachment was accompanied by the formation of spheroid-shaped cell clusters between days 13-15. These cell aggregates formed in multiple layers, with detached cells on the top surface and attached cells at the peripheral edges being elongated with diminished intercellular connections. Also, the remaining attached cells developed marked cytoplasmic granules and vacuolization. These behaviors are notable deviations from normal Vero cell growth with media change (CG group herein), as this cell type typically grows as confluent monolayers and does not normally exhibit intracellular granulation or vacuolization. The observation that the Vero cells lost substrate adherence, however, is not a unique growth characteristic of this cells type or other cultured epithelial cell types. Substrate adhesion is facilitated by the establishment of critical components of the extracellular matrix (ECM). Loss of substrate adherence may be due to aberrant ECM synthesis which is an indication of enervated cell health. ${ }^{10,15}$ The additional finding that the EG group was the only group to possess viable detached cells in its supernatant at day 15 , however, does lend itself to support the postulate that nutritional stress and overcrowding were potential factors in the cell detachment of this group.

It is apparent that Vero cells grown in the presence of media without glucose and without media change, the ENG group, is lethal by 15 days. It is unclear as to why the cells deprived of glucose exhibit an elongated and abnormal shape change by day 8 in culture, but it is speculated that these cells are stretching to create cell to cell connections due to their less confluent nature. Indeed, as the confluency decreased the stretching of the cells became more evident, and this group never gained complete confluency during its nine days of culture.

Altered cell morphology and growth characteristics of the EG cells persisted after re-plating of the detached, floating cells and spheroid clusters that were centrifuged and re-suspended into fresh media at day 15. Moreover, abnormal EG cell morphology progressed further after re-plating. Augmented stress-induced morphological changes included spindle-like cytoplasmic extensions, degenerated intercellular associations, and plasmolytic shape. Additionally, these cells developed enhanced cytoplasmic granulation and vacuolization as compared to observations made before passage.

As previously noted, conditions of nutritional stress in Vero cells have been previously shown to induce deviant growth characteristics. ${ }^{10}$ Detachment of cells and spheroid formation has been explained as a loss of contact inhibition. This may also explain why the EG cells detached from their cell culture monolayer and were viable, while few control CG cells detached and those that did were non-viable. Prior work has documented successful culturing of nutritionally stressed detached Vero cells in soft agar medium. Such behavior has been interpreted as "anchorage independence," a characteristic which is deviant from standard Vero cell growth and commonly seen in malignancy. ${ }^{10,15}$

While loss of contact inhibition has been identified as a plausible underlying cellular process which promoted cell detachment and spheroid formation, other plausible processes include alterations in gene expression and regulation, cytoskeletal dynamics, extracellular matrix deposition and integrity, and/or second messenger systems - all of which are yet to be fully elucidated. Noteworthy is the work of Smets (1980) who associated cellular transformation with serial modifications of the cellular genome, resulting in irregular gene expression. ${ }^{3}$ Specifically, Genari et al. (1998) identified $44 \mathrm{kDa}$ and $78 \mathrm{kDa}$ proteins in nutritionally-stressed Vero cells as potential heat shock proteins (HSPs). ${ }^{15}$ The role of these two suspected HSPs is speculated to aid in sustaining the altered Vero cell phenotype. This was observed in conjunction with aberrant fibronectin expression in Vero cells that underwent cell transformation under nutritional stress conditions. ${ }^{10}$ Fibronectin 
is an essential component of the ECM. Previous work by Chakrabarty et al. (1997) explained that the ECM modulates cellular processes such as cell proliferation and gene expression. ${ }^{16}$ Mechanical stimuli transmitted through the ECM can mobilize intracellular signal cascades to affect gene expression..$^{17}$ Indeed, abrogated fibronectin is a trademark of epithelial tumor development. ${ }^{16}$ Interestingly, transformed chick embryo fibroblasts have been used as a model for the return of normal morphology, surface adhesion, and contact inhibition when the expression of major glycoproteins are restored. ${ }^{18}$ This further demonstrates the importance of the extracellular matrix in normal mammalian cell growth and the potential effects of cellular crowding on altering gene expression.

In summary, the results presented here confirm the essential roles that media change and sub-culturing procedures play in maintaining the morphological integrity of Vero cells, as shown previously by Genari et al. (1998)..$^{10}$ These results also demonstrate the utility of Vero cells as a model to study cell transformation induced by artificially implemented nutritional stress paradigms in vitro. As such, subsequent undergraduate research experiments that employ the Vero cell model can be used to further investigate the cumulative culture conditions that induced the changes observed in this paper. Before and after the fifteen day culture period, $\mathrm{pH}$, osmolarity, and glucose concentration measurements of the all culture conditions must be explicitly determined and compared, especially to those of the control group. This would provide insight into which stressors are most important in the transformation process. Indeed, little is known on the effect of glucose deprivation in transforming cell morphology. Some other unanswered questions include: What metabolic fuel source is relied upon by nutritionally-stressed and overcrowded Vero cells; How does ECM synthesis change as cells transition in detached and/or spheroid clusters; Are the transformation-like Vero cell morphology and growth changes truly analogous to neoplastic transformation processes at the genomic level, and if so in what ways? Subsequent experiments will also include other mammalian, adherent cell lines to determine if similar results can be generated in different cell types.

\section{ACKNOWLEDGMENTS}

We thank Penn State Lehigh Valley and Olympus for resource provisions. 


\section{REFERENCES}

[1] Genari, S., and Wada, M. (1995) Behavioral differences and cytogenic analysis of a transformed cellular population derived from a Vero cell line, Cytobioas 81, 17-25.

[2] Alberts, B., Johnson, A., Lewis, J., Raff, M., Roberts, K., and Walter, P. (2008) Cancer, In Molecular Biology of the Cell, pp 1209-1215, Garland Science, New York, NY.

[3] Smets, L. (1980) Cell transformation as a model for tumor induction and neoplastic growth, Biochemica et Biophysica Acta 605, 93-111.

[4] Keski-Oja, J., Alitalo, K., Barlati, S., and Vaheri, A. (1985) Pericellular matrix changes in fibroblastic and epithelial cells induced by oncogenic transformation, In Theories and Models in Cell Transformation (Tumori, I. S., Ed.), pp 55-70, Academic Press, Genoa, Italy.

[5] Nermut, M. V., Eason, P., Hirst, E. M., and Kellie, S. (1991) Cell/substratum adhesions in RSV-transformed rat fibroblasts, Exp Cell Res 193, 382-397.

[6] Vasiliev, J. M. (1985) Spreading of non-transformed and transformed cells, Biochimica et biophysica acta 780, 21-65.

[7] Group, I. N. E. W. (1985) Cellular and molecular mechanisms of cell transformation and standardization of transformation assays of established cell lines for the prediction of carcinogenic chemicals; overview and recommended protocols, Cancer Research 45, 2395-2399.

[8] Pónten, J. (1976) The relationship between in vitro transformation and tumor formation in vivo, Biochemica et Biophysica Acta 458, 397-422.

[9] Terzaghi-Howe, M. (1993) Factors regulating the emergence of spontaneous and X-ray-induced variants in primary rat tracheal epithelial cell cultures, In vitro cellular \& developmental biology: journal of the Tissue Culture Association 29A, 120-126.

[10] Genari, S. C., Gomes, L., and Wada, M. L. (1998) Alterations in the growth and adhesion pattern of Vero cells induced by nutritional stress conditions, Cell biology international 22, 285-294.

[11] Berenblum, I., and Armuth, V. (1981) Two independent aspects of tumor promotion, Biochimica et biophysica acta 651, 51-63.

[12] Genari, S. C., Dolder, M. A., and Wada, M. L. (1996) Scanning and transmission electron microscopy of transformed Vero cells, with altered in vitro growth characteristics, Journal of submicroscopic cytology and pathology 28, 565-572.

[13] Landry, J., Bernier, D., Ouellet, C., Goyette, R., and Marceau, N. (1985) Spheroidal aggregate culture of rat liver cells: histotypic reorganization, biomatrix deposition, and maintenance of functional activities, J Cell Biol 101, 914-923.

[14] Takezawa, T., Mori, Y., Yonaha, T., and Yoshizato, K. (1993) Characterization of morphology and cellular metabolism during the spheroid formation by fibroblasts, Exp Cell Res 208, 430-441.

[15] Freshney, I. (2011) In Culture of Animal Cells: A Manual of Basic Technique and Specialized Applications Sixth Edition ed., John Wiley \& Sons, Inc., Hoboken, NJ.

[16] Chakrabarty, S., Reynolds, S., Wang, H., and Rajagopal, S. (1997) Action of polypeptide growth factors in colon cancer: Development of new therapeutic approaches, In Frontiers in Bioscience 2.

[17] Alberts, B., Bray, D., Hopkin, K., Johnson, A., Lewis, J., Raff, M., K, R., and Walter, P. (2010) Cellular Communities: Tissues, Stem Cells, and Cancer, In Essential Cell Biology, pp 696-697, Garland Science, New York, NY.

[18] Yamada, K. M., Yamada, S. S., and Pastan, I. (1976) Cell surface protein partially restores morphology, adhesiveness, and contact inhibition of movement to transformed fibroblasts, Proc Natl Acad Sci USA 73, 1217-1221. 


\section{PRESS SUMMARY}

Vero cells were monitored for nine and fifteen day periods under nutritional deprivation and over-crowding conditions. The stressed cells remained viable and underwent a transformation in growth patterns and morphology analogous to behavior seen other models of mammalian cell transformation. These alterations were maintained in the stressed cells after re-plating, suggesting that the transformation was permanent. The results suggest that deprivation of nutrition and other factors essential to life may facilitate aberrant growth.

\section{ABOUT THE STUDENT AUTHORS}

Tyler Adams is currently a third year student at Penn State Berks. He is pursuing a B.S. in Biology. After graduation, he intends on earning his M.D. and specializing in cardiology.

Rabia Anwar is a third year student at Penn State Berks. She is pursuing a B.S. in Genetics and Developmental Biology and a minor in Entrepreneurship and Innovation. After graduation, she intends on earning her M.D. and specializing in cardiology.

Michael Mfarej is currently a fourth year Penn State Berks student. He is pursuing a B.S. in Genetics and Developmental Biology. He will be graduating in the Spring of 2015. After graduation, he plans to pursue a Ph.D. in Cellular and Molecular Biology with interests of continuing his research training in cancer biology.

Taylor Rundatz is currently a fourth year Penn State Berks student. She intends on graduating in the Spring of 2015 with a B.S. in Biology. After graduation, she plans to pursue an M.D. and Ph.D. dual degree program. With her medical education she is interested in a career as a pathologist. 